\title{
Global Asymptotic Stabilization Control for a Class of Nonlinear Systems with Dynamic Uncertainties
}

\author{
Jiangbo Yu, ${ }^{1}$ Jizhong Wang, ${ }^{1}$ and Zhongcai Zhang ${ }^{2}$ \\ ${ }^{1}$ School of Science, Shandong Jianzhu University, Jinan 250101, China \\ ${ }^{2}$ School of Automation, Southeast University, Nanjing 210096, China \\ Correspondence should be addressed to Jiangbo Yu; jbyu2002@163.com
}

Received 11 May 2015; Accepted 16 August 2015

Academic Editor: Uchechukwu E. Vincent

Copyright (C) 2015 Jiangbo Yu et al. This is an open access article distributed under the Creative Commons Attribution License, which permits unrestricted use, distribution, and reproduction in any medium, provided the original work is properly cited.

\begin{abstract}
This paper is concerned with the global asymptotic stabilization control problem for a class of nonlinear systems with input-to-state stable (ISS) dynamic uncertainties and uncertain time-varying control coefficients. Unlike the existing works, the ISS dynamic uncertainty is characterized by the uncertain supply rates. By using the backstepping control approach, a systematic controller design procedure is developed. The designed control law can guarantee that the system states are asymptotically regulated to the origin from any initial conditions and the other signals are bounded in closed-loop systems. Moreover, it is shown that, under some additional conditions, a linear control law can be designed by the proposed methodology. The simulation example demonstrates its effectiveness.
\end{abstract}

\section{Introduction}

The nonlinear control theory is an active research direction in the control field because of its widespread applications in the real world. During the past two decades, various novel methodologies have been generated for the nonlinear feedback control; see the recent survey [1] and references therein for an interesting introduction to this area. One of the influential notions is the input-to-state stability (ISS) and its several variants. Since they are introduced by Sontag in $[2,3]$, the notion of ISS as well as its integral variant-integral ISS (iISS)-has become a foundational concept upon which much of modern nonlinear feedback analysis and design rest. As noted in [4], ISS provides a nonlinear generalization of finite gains with respect to supremum norms and also of finite $L^{2}$ gains, and it plays a central role in recursive design, coprime factorizations, controllers for nonminimum phase systems, and many other areas. Based on the series of works on ISS, the nonlinear small-gain theorem was proposed in the state-space setting and is widely used in the stability analysis and control design for complex interconnected systems in [5]. The stochastic results can be found in $[6,7]$ and the references therein.

It is noted that a unifying framework is presented in [8] for the global output feedback regulation control problem from ISS to iISS. The framework established in [8] extends many known classes of output feedback form systems. However, the system uncertainties investigated there depend only on the system output and the inverse system state. With unmeasured states dependent growth, in $[9,10]$, the problem of global stabilization by output/state feedback is investigated for a class of nonlinear systems with uncertain control coefficients. However, there is no dynamic uncertainty for the system under consideration. In [11], this work is further studied for a larger class of nonlinear uncertain systems, in which the observer gain is governed by a Riccati differential equation. Moreover, the output regulation problem is also considered in [12] for this class of nonlinear systems with iISS inverse dynamics. Later, in $[13,14]$, this work is further investigated for the nonlinear systems with uncertain nonlinearities dependent on all unmeasured states. However, the control coefficients in above results are required to be known a priori or unknown nonzero constants. In [15], the global set-point tracking control is investigated for a class of cascaded nonlinear systems with unknown control coefficients. However, a restrictive condition is that the control coefficients are required to have the same signs.

In this paper, we will further investigate this problem for a class of nonlinear systems with more general nonlinear 
uncertainties. Unlike the existing works such as in $[9,12,15-$ 17], the studied system is with the uncertain control coefficients, which could be unknown time-varying functions. Another feature of this work is that the dynamic uncertainties are characterized by the uncertain ISS supply rates. This is different from the existing results reported in literatures where the ISS dynamic uncertainty is investigated under the hypothesis that supply rates are known a priori such as in $[8$, $11,13,15]$. With the help of the backstepping approach [18], we design a robust adaptive controller which could achieve the system states convergent to the origin while the other signals are bounded. Moreover, it is of interest to note that a linear control law can be designed using the developed scheme if some stronger conditions are imposed on the nonlinear system.

The rest of the paper is organized as follows. In Section 2, we provide some mathematical preliminaries and state the problem. The controller design procedure is developed in Section 3, and the main result is presented in Section 4. Section 5 illustrates the obtained results by a numerical example. Section 6 concludes this paper.

Notation. Let $\mathbf{R}\left(\mathbf{R}_{+}\right)$denote the set of all (positive) real numbers and let $R^{\mathbf{n}}$ denote the real $n$-dimensional space. For a given vector or matrix $X, X^{T}$ denotes its transpose. For any column vector $x=\left(x_{1}, \ldots, x_{n}\right)^{T} \in \mathbf{R}^{\mathbf{n}}, \bar{x}_{i}$ denotes the column vector consisting of the first $i$ elements of $x$ in the original order; that is, $\bar{x}_{i}=\left(x_{1}, \ldots, x_{i}\right)^{T}$. Specifically, for $x=\left(x_{1}, \ldots, x_{n}\right)^{T}, x_{1}=\bar{x}_{1}, x=\bar{x}_{n}$. A continuous function $\alpha:[0, a) \rightarrow[0, \infty)$ is said to belong to class- $K$ if it is strictly increasing and $\alpha(0)=0$. It is said to belong to class$K_{\infty}$ if $a=\infty$ and $\alpha(r) \rightarrow \infty$ as $r \rightarrow \infty$. The notation $\widetilde{\beta}(s)=O(\beta(s))$ means that there exist two positive constants $k$ and $c$ such that $\widetilde{\beta}(s) \leq k \beta(s), \forall|s|<c$.

\section{Problem Formulation}

In this paper, we consider the following class of cascaded nonlinear systems with dynamic uncertainties:

$$
\begin{gathered}
\dot{\eta}=q(t, \eta, y), \\
\dot{x}_{1}=d_{1}(t) x_{2}+g_{1}(t, \eta, x), \\
\vdots \\
\dot{x}_{n}=d_{n}(t) u+g_{n}(t, \eta, x), \\
y=x_{1},
\end{gathered}
$$

where $u \in \mathbf{R}$ is the control input, $y \in \mathbf{R}$ is the system output, $\boldsymbol{x}=\left(x_{1}, \ldots, x_{n}\right) \in \mathbf{R}^{\mathbf{n}}$ are the system states, and $\eta \in \mathbf{R}^{\mathbf{r}}$ is referred to as dynamic uncertainties, which is unmeasured and hence is not available for feedback design. The continuous functions $d_{i}(t)(i=1, \ldots, n)$ called the control coefficients are assumed to be unknown; particularly, $d_{i}(t) \neq 0$; the unmodeled (or uncertain) dynamics $q(\cdot)$ and $g_{i}(\cdot)(i=1, \ldots, n)$ are locally Lipschitz.
The control objective in this paper is to find a smooth, dynamic, partial-state feedback law of the form

$$
\begin{aligned}
& \dot{\xi}=\chi_{\xi}(\xi, x), \\
& u=\chi_{u}(\xi, x),
\end{aligned}
$$

where $\chi_{\xi}$ and $\chi_{u}$ are smooth functions such that all solutions $(\eta(t), x(t), \xi(t))$ in closed-loop system are bounded on $[0, \infty)$ and specially the system states $(\eta(t), x(t))$ asymptotically converge to the origin. Toward this end, throughout the paper, we make the following assumptions on system (1).

Assumption 1. The $\eta$-subsystem is input-to-state stable (ISS) with state $\eta$ and input $y$; that is, there exists a positive-definite and proper ISS-Lyapunov function $V_{0}$, such that

$$
\begin{gathered}
\underline{\alpha}(|\eta|) \leq V_{0}(\eta) \leq \bar{\alpha}(|\eta|), \\
\frac{\partial V_{0}}{\partial \eta}(\eta) q(t, \eta, y) \leq-\alpha_{0}(|\eta|)+\delta_{0} \gamma_{0}(|y|),
\end{gathered}
$$

where $\underline{\alpha}(\cdot), \bar{\alpha}(\cdot), \alpha_{0}(\cdot), \gamma_{0}(\cdot) \in K_{\infty}$ and $\delta_{0}>0$ is an unknown constant.

Remark 2. According to [19], one knows that $\eta$-subsystem satisfying (3) is ISS, and the function pair $\left(\alpha_{0}, \delta_{0} \gamma_{0}\right)$ is viewed as the supply rates. Since $\delta_{0}$ in (3) is unknown, the dynamic uncertainty has uncertain ISS supply rates. This is different from the existing results reported in literatures, where the ISS dynamic uncertainty is investigated with the supply rates assumed to be known a priori, such as $[8,11,13,15]$.

Assumption 3. For the uncertain nonlinearities $g_{i}(t, \eta, x)(i=$ $1, \ldots, n)$, there exist unknown positive constants $p_{i j}(i=$ $1, \ldots, n ; j=1,2)$ such that

$$
\begin{array}{r}
\left|g_{i}(t, \eta, x)\right| \leq p_{i 1} \phi_{i 1}(|\eta|)+p_{i 2} \phi_{i 2}\left(\left|x_{1}, \ldots, x_{i}\right|\right), \\
i=1, \ldots, n,
\end{array}
$$

where $\phi_{i j}(\cdot)$ are known smooth functions and $\phi_{i j}(0)=0(i=$ $1, \ldots, n ; j=1,2)$.

Assumption 4. There exist known positive constants $\underline{d}_{i}$ and $\bar{d}_{i}(i=1, \ldots, n)$, such that

$$
0<\underline{d}_{i} \leq d_{i}(t) \leq \bar{d}_{i}, \quad i=1, \ldots, n .
$$

To deal with the unmeasured state $\eta$, we have the following lemma, which plays an important role in the coming feedback design and stability analysis.

Lemma 5. Consider the $\eta$-subsystem satisfying Assumption 1. Suppose

$$
\gamma_{0}(s)=O\left(s^{2}\right)
$$

and then we can choose a positive continuous function $\rho(\cdot)$, such that the function

$$
\bar{V}_{0}(\eta)=\int_{0}^{V_{0}(\eta)} \rho(s) d s
$$


is another candidate ISS-Lyapunov function satisfying

$$
\begin{aligned}
\frac{\partial \bar{V}_{0}}{\partial \eta}(\eta) q(t, \eta, y) \leq & -(1-\epsilon) \rho \circ \underline{\alpha}(|\eta|) \alpha_{0}(|\eta|) \\
& +\delta \gamma(|y|),
\end{aligned}
$$

where $\epsilon(0<\epsilon<1)$ is a small design constant, $\delta>0$ is an unknown constant, and $\gamma$ is a $K_{\infty}$-function with $\gamma(s)=O\left(s^{2}\right)$.

Remark 6. If $\gamma(s)=O\left(s^{2}\right)$, according to Lemma 2 in [19], there exists a smooth nondecreasing function $\bar{\gamma}(\cdot)$ satisfying

$$
\gamma(s) \leq \bar{\gamma}(s) s^{2}
$$

Lemma 7. For any $C^{1}$ function $f\left(x_{1}, \ldots, x_{n}\right)$, there exist continuous functions $f_{i}\left(x_{1}, \ldots, x_{n}\right)(1 \leq i \leq n)$, such that

$$
f\left(x_{1}, \ldots, x_{n}\right)=f(0, \ldots, 0)+\sum_{i=1}^{n} x_{i} f_{i}\left(x_{1}, \ldots, x_{n}\right) .
$$

Remark 8. According to Lemma 7, from $\phi_{i 2}(0)=0(i=$ $1, \ldots, n)$ in Assumption 3, it is known that, for each $\phi_{i 2}(\cdot)$, there exist smooth functions $\phi_{i 2 j}(\cdot)(1 \leq j \leq i)$ satisfying

$$
\phi_{i 2}\left(x_{1}, \ldots, x_{i}\right)=\sum_{j=1}^{i} x_{j} \phi_{i 2 j}\left(x_{1}, \ldots, x_{i}\right), \quad i=1, \ldots, n \text {. }
$$

\section{Controller Design}

In this section, we give the controller design procedure using the backstepping design method.

Step 1. Starting with the $x_{1}$-subsystem $\dot{x}_{1}=d_{1}(t) x_{2}+g_{1}(t, \eta$, $x$ ). We consider the variable $x_{2}$ as the virtual control input. Let $z_{1}=x_{1}$ and $z_{2}=x_{2}-\vartheta_{1}$ where $\vartheta_{1}$ is the intermediate control input. Considering Lemma 5 and Remark 6, along solutions of (1), the time derivative of the function

$$
V_{1}=\frac{1}{2} z_{1}^{2}+\bar{V}_{0}(\eta)
$$

satisfies

$$
\begin{aligned}
\dot{V}_{1} \leq & z_{1}\left(d_{1}(t) \vartheta_{1}+g_{1}(t, \eta, x)\right)+d_{1}(t) z_{1} z_{2} \\
& -(1-\epsilon) \rho \circ \underline{\alpha}(|\eta|) \alpha_{0}(|\eta|)+\delta \bar{\gamma}\left(\left|x_{1}\right|\right) x_{1}^{2} .
\end{aligned}
$$

According to Assumption 3 and the completion of squares, we have

$$
\begin{gathered}
z_{1} g_{1}(t, \eta, x) \leq\left|z_{1}\right|\left(p_{11} \phi_{11}(|\eta|)+p_{12}\left|z_{1}\right| \phi_{121}\left(x_{1}\right)\right) \\
\leq \phi_{11}^{2}(|\eta|)+\frac{1}{4} z_{1}^{2} p_{11}^{2} \\
+z_{1}^{2} p_{12}\left|\phi_{121}\left(x_{1}\right)\right| .
\end{gathered}
$$

Define $p^{*}=\max \left\{\delta, p_{i 1}, p_{i 2}, p_{i 1}^{2}, p_{i 2}^{2} \mid i=1, \ldots, n\right\}$, and we get

$$
\begin{aligned}
& z_{1} g_{1}(t, \eta, x)+\delta \bar{\gamma}\left(\left|x_{1}\right|\right) x_{1}^{2} \\
& \leq \phi_{11}^{2}(|\eta|)+\frac{1}{4} z_{1}^{2} p_{11}{ }^{2}+z_{1}^{2} p_{12}\left|\phi_{121}\left(x_{1}\right)\right| \\
& \quad+\delta \bar{\gamma}\left(\left|x_{1}\right|\right) x_{1}^{2} \\
& \leq \phi_{11}^{2}(|\eta|)+p^{*} z_{1}^{2}\left(\frac{1}{4}+\left|\phi_{121}\left(x_{1}\right)\right|+\bar{\gamma}\left(\left|x_{1}\right|\right)\right) \\
& \leq \phi_{11}^{2}(|\eta|)+z_{1}^{2} \widehat{\phi}_{1}\left(x_{1}\right) p^{*}
\end{aligned}
$$

with a new smooth function $\widehat{\phi}_{1}\left(x_{1}\right) \geq 1 / 4+\left|\phi_{121}\left(x_{1}\right)\right|+$ $\bar{\gamma}\left(\left|x_{1}\right|\right)>0$. As a result, there holds

$$
\begin{aligned}
\dot{V}_{1} \leq & -(1-\epsilon) \rho \circ \underline{\alpha}(|\eta|) \alpha_{0}(|\eta|)+d_{1}(t) z_{1} \vartheta_{1} \\
& +d_{1}(t) z_{1} z_{2}+\phi_{11}^{2}(|\eta|)+z_{1}^{2} \widehat{\phi}_{1}\left(x_{1}\right) p^{*}
\end{aligned}
$$

Considering the unknown constant $p^{*}$ in (16), we use an adaptive signal $\widehat{p}$ to estimate $p^{*}$. Consequently, we augment $V_{1}$ with the parameter estimation error $\widetilde{p}=p^{*}-\widehat{p}$, such as

$$
\bar{V}_{1}=V_{1}+\frac{1}{2 \Upsilon} \widetilde{p}^{2}
$$

where $\Upsilon>0$ is the design parameter. In view of $(16)$ and $p^{*}=$ $\widehat{p}+\widetilde{p}$, a direct substitution leads to

$$
\begin{aligned}
\dot{\bar{V}}_{1} \leq & -(1-\epsilon) \rho \circ \underline{\alpha}(|\eta|) \alpha_{0}(|\eta|)+d_{1}(t) z_{1} \vartheta_{1} \\
& +z_{1}^{2} \widehat{\phi}_{1}\left(x_{1}\right) \widehat{p}+d_{1}(t) z_{1} z_{2}+\phi_{11}^{2}(|\eta|) \\
& +\frac{1}{\Upsilon} \widetilde{p}\left(\Upsilon \widehat{\phi}_{1}\left(x_{1}\right) z_{1}^{2}-\dot{\hat{p}}\right) .
\end{aligned}
$$

Considering Assumption 4, we take the virtual control

$$
\vartheta_{1}=-\frac{1}{\underline{d}_{1}}\left(\nu_{1}+\widehat{\phi}_{1}\left(x_{1}\right) \hat{p}\right) z_{1}
$$

where $v_{1}>0$ is a design constant to be determined later. Let

$$
\tau_{1}=\Upsilon \widehat{\phi}_{1}\left(x_{1}\right) z_{1}^{2}
$$

and then we get

$$
\begin{aligned}
\dot{\bar{V}}_{1} \leq & -(1-\epsilon) \rho \circ \underline{\alpha}(|\eta|) \alpha_{0}(|\eta|)-\nu_{1} z_{1}^{2}+\phi_{11}^{2}(|\eta|) \\
& +d_{1}(t) z_{1} z_{2}+\frac{1}{\Upsilon} \widetilde{p}\left(\tau_{1}-\dot{\hat{p}}\right) .
\end{aligned}
$$

Remark 9. It is noted that, in (19), we assume that $\widehat{p}(t) \geq 0$. In fact, from the updating law of $\dot{\hat{p}}$ given later, this property can be guaranteed by choosing the initial condition $\vec{p}(0) \geq 0$. Alternatively, using the idea in [20], we also can apply the $\widehat{p} \leq$ $\sqrt{1+\widehat{p}^{2}}$ or $\widehat{p} \leq\left(1+\widehat{p}^{2}\right) / 2$ instead of $\widehat{p}$. 
Step 2. Let $z_{3}=x_{3}-\vartheta_{2}$, where $\vartheta_{2}$ is the virtual control law. We consider the Lyapunov function

$$
V_{2}=\bar{V}_{1}+\frac{1}{2} z_{2}^{2}
$$

In view of (21), we have

$$
\begin{aligned}
\dot{V}_{2} & \leq-(1-\epsilon) \rho \circ \underline{\alpha}(|\eta|) \alpha_{0}(|\eta|)-v_{1} z_{1}^{2}+\phi_{11}^{2}(|\eta|) \\
& +d_{2}(t) z_{2} z_{3}+\frac{1}{\Upsilon} \tilde{p}\left(\tau_{1}-\dot{\hat{p}}\right)+z_{2}\left(d_{2}(t) \vartheta_{2}\right. \\
& +g_{2}(t, \eta, x)-\frac{\partial \vartheta_{1}}{\partial x_{1}} g_{1}(t, \eta, x)+d_{1}(t) z_{1} \\
& \left.-\frac{\partial \vartheta_{1}}{\partial x_{1}} d_{1}(t) x_{2}-\frac{\partial \vartheta_{1}}{\partial \widehat{p}} \dot{\hat{p}}\right) .
\end{aligned}
$$

From (4) and (11), the following calculations hold:

$$
\begin{aligned}
z_{2} g_{2}(t, \eta, x) \leq & \left|z_{2}\right| p_{21} \phi_{21}(|\eta|) \\
& +\left|z_{2}\right| p_{22}\left|x_{1}\right| \phi_{121}\left(\bar{x}_{2}\right) \\
& +\left|z_{2}\right| p_{22}\left(\left|z_{2}+\vartheta_{1}\right|\right) \phi_{122}\left(\bar{x}_{2}\right) .
\end{aligned}
$$

Like the calculations in (14), by completing the squares, we have

$$
\begin{aligned}
- & z_{2} \frac{\partial \vartheta_{1}}{\partial x_{1}} g_{1}(t, \eta, x) \\
\leq & \phi_{11}^{2}(|\eta|)+z_{1}^{2} \\
& +p^{*} z_{2}^{2}\left(\frac{1}{4}\left(\frac{\partial \vartheta_{1}}{\partial x_{1}}\right)^{2}+\frac{1}{4}\left(\frac{\partial \vartheta_{1}}{\partial x_{1}}\right)^{2} \phi_{121}^{2}\left(x_{1}\right)\right) .
\end{aligned}
$$

Define $\widehat{\phi}_{21}\left(x_{1}, \widehat{p}\right)=(1 / 4)\left(\partial \vartheta_{1} / \partial x_{1}\right)^{2}\left(1+\phi_{121}^{2}\left(x_{1}\right)\right)$, and then we have

$$
\begin{aligned}
-z_{2} \frac{\partial \vartheta_{1}}{\partial x_{1}} g_{1}(t, \eta, x) \leq & \phi_{11}^{2}(|\eta|)+z_{1}^{2} \\
& +z_{2}^{2} \widehat{\phi}_{21}\left(x_{1}, \widehat{p}\right) p^{*}
\end{aligned}
$$

In the same manner, using the completion of squares again, it can be verified that

$$
\begin{aligned}
& \left|z_{2}\right| p_{21} \phi_{21}(|\eta|) \leq \phi_{21}^{2}(|\eta|)+\frac{1}{4} p_{21}^{2} z_{2}^{2} \\
& \left|z_{2}\right| p_{22}\left|z_{1}\right| \phi_{121}\left(\bar{x}_{2}\right) \leq z_{1}^{2}+\frac{1}{4} p_{22}^{2} z_{2}^{2} \phi_{121}^{2}\left(\bar{x}_{2}\right) \\
& \left|z_{2}\right| p_{22}\left(\left|z_{2}+\vartheta_{1}\right|\right) \phi_{122}\left(\bar{x}_{2}\right) \\
& \leq z_{2}^{2} p_{22} \phi_{122}\left(\bar{x}_{2}\right)+\left|z_{2}\right| p_{22}\left|\vartheta_{1}\right| \phi_{122}\left(\bar{x}_{2}\right) \\
& \leq z_{2}^{2} p_{22} \phi_{122}\left(\bar{x}_{2}\right) \\
& \quad+\left|z_{2}\right| p_{22}\left|-\frac{1}{\underline{d}_{1}}\left(\nu_{1}+\widehat{\phi}_{1}\left(x_{1}\right) \hat{p}\right) z_{1}\right| \phi_{122}\left(\bar{x}_{2}\right)
\end{aligned}
$$

$$
\begin{aligned}
\leq & z_{2}^{2} p_{22} \phi_{122}\left(\bar{x}_{2}\right)+z_{1}^{2} \\
& +\frac{1}{4} p_{22}^{2} z_{2}^{2} \frac{1}{\underline{d}_{1}^{2}}\left(\nu_{1}+\widehat{\phi}_{1}\left(x_{1}\right) \hat{p}\right)^{2} \phi_{122}^{2}\left(\bar{x}_{2}\right) .
\end{aligned}
$$

As a result,

$$
\begin{aligned}
& z_{2} g_{2}(t, \eta, x) \leq \phi_{21}^{2}(|\eta|)+2 z_{1}^{2}+z_{2}^{2} p^{*}\left(\frac{1}{4}\right. \\
& +\frac{1}{4} \phi_{121}^{2}\left(\bar{x}_{2}\right)+\phi_{122}\left(\bar{x}_{2}\right) \\
& \left.+\frac{1}{4} \frac{1}{\underline{d}_{1}^{2}}\left(v_{1}+\widehat{\phi}_{1}\left(x_{1}\right) \hat{p}\right)^{2} \phi_{122}^{2}\left(\bar{x}_{2}\right)\right)
\end{aligned}
$$

Define $\widehat{\phi}_{22}\left(\bar{x}_{2}, \widehat{p}\right)=1 / 4+(1 / 4) \phi_{121}^{2}\left(\bar{x}_{2}\right)+\phi_{122}\left(\bar{x}_{2}\right)+(1 / 4)(1 /$ $\left.\underline{d}_{1}^{2}\right)\left(\nu_{1}+\widehat{\phi}_{1}\left(x_{1}\right) \widehat{p}\right)^{2} \phi_{122}^{2}\left(\bar{x}_{2}\right)$, and then

$$
z_{2} g_{2}(t, \eta, x) \leq \phi_{21}^{2}(|\eta|)+2 z_{1}^{2}+z_{2}^{2} p^{*} \widehat{\phi}_{22}\left(\bar{x}_{2}, \widehat{p}\right) .
$$

Let $\widehat{\phi}_{2}\left(\bar{x}_{2}, \widehat{p}\right)=\widehat{\phi}_{21}\left(x_{1}, \widehat{p}\right)+\widehat{\phi}_{22}\left(\bar{x}_{2}, \widehat{p}\right)>0$, and then we get

$$
\begin{aligned}
& z_{2}\left(g_{2}(t, \eta, x)-\frac{\partial \vartheta_{1}}{\partial x_{1}} g_{1}(t, \eta, x)\right) \\
& \quad \leq \sum_{j=1}^{2} \phi_{j 1}^{2}(|\eta|)+3 z_{1}^{2}+z_{2}^{2} \widehat{\phi}_{2}\left(\bar{x}_{2}, \widehat{p}\right) p^{*}
\end{aligned}
$$

From Assumption 4, it is deduced that

$$
\begin{gathered}
z_{2}\left(d_{1}(t) z_{1}-\frac{\partial \vartheta_{1}}{\partial x_{1}} d_{1}(t) x_{2}\right) \\
\leq\left|z_{2}\right|\left|d_{1}(t)\right|\left|z_{1}\right|+\left|z_{2}\right|\left|\frac{\partial \vartheta_{1}}{\partial x_{1}}\right|\left|d_{1}(t)\right|\left(\left|z_{2}\right|+\left|\vartheta_{1}\right|\right) \\
\leq z_{1}^{2}+\frac{\bar{d}_{1}^{2}}{4} z_{2}^{2}+z_{2}^{2} \frac{\bar{d}_{1}}{2}\left(1+\left(\frac{\partial \vartheta_{1}}{\partial x_{1}}\right)^{2}\right)+z_{1}^{2} \\
+\frac{1}{4} z_{2}^{2}\left(\frac{\partial \vartheta_{1}}{\partial x_{1}}\right)^{2} \frac{\bar{d}_{1}^{2}}{\underline{d}_{1}^{2}}\left(\nu_{1}+\widehat{\phi}_{1}\left(x_{1}\right) \widehat{p}\right)^{2}
\end{gathered}
$$

Define $\bar{\phi}_{21}\left(\bar{x}_{2}, \widehat{p}\right)=\bar{d}_{1}^{2} / 4+(1 / 4)\left(\bar{d}_{1}^{2} / \underline{d}_{1}^{2}\right)\left(\partial \vartheta_{1} / \partial x_{1}\right)^{2}\left(\nu_{1}+\right.$ $\left.\widehat{\phi}_{1}\left(x_{1}\right) \widehat{p}\right)^{2}+\left(\bar{d}_{1} / 2\right)\left(1+\left(\partial \vartheta_{1} / \partial x_{1}\right)^{2}\right)$, and there holds

$$
z_{2}\left(d_{1}(t) z_{1}-\frac{\partial \vartheta_{1}}{\partial x_{1}} d_{1}(t) x_{2}\right) \leq 2 z_{1}^{2}+z_{2}^{2} \bar{\phi}_{21}\left(\bar{x}_{2}, \widehat{p}\right)
$$

Take the following notation:

$$
\tau_{2}=\tau_{1}+\Upsilon z_{2}^{2} \widehat{\phi}_{2}\left(\bar{x}_{2}, \widehat{p}\right)
$$


and furthermore, in view of (30) and (32), we obtain

$$
\begin{aligned}
\dot{V}_{2} & \leq-(1-\epsilon) \rho \circ \underline{\alpha}(|\eta|) \alpha_{0}(|\eta|)-\left(\nu_{1}-5\right) z_{1}^{2} \\
& +2 \phi_{11}^{2}(|\eta|)+\phi_{21}^{2}(|\eta|)+d_{2}(t) z_{2} z_{3}+z_{2}\left(d_{2}(t) \vartheta_{2}\right. \\
& \left.+z_{2} \widehat{\phi}_{2}\left(\bar{x}_{2}, \widehat{p}\right) \hat{p}+z_{2} \bar{\phi}_{21}\left(\bar{x}_{2}, \widehat{p}\right)-\frac{\partial \vartheta_{1}}{\partial \widehat{p}} \dot{\hat{p}}\right)+\frac{1}{\Upsilon} \\
& \cdot \tilde{p}\left(\tau_{2}-\dot{\hat{p}}\right) .
\end{aligned}
$$

Considering $d_{2}(t)$ is unknown, the term of $-\left(\partial \vartheta_{1} / \partial \widehat{p}\right) \dot{\hat{p}}$ can not be canceled. In fact, we express (34) as

$$
\begin{aligned}
\dot{V}_{2} & \leq-(1-\epsilon) \rho \circ \underline{\alpha}(|\eta|) \alpha_{0}(|\eta|)-\left(\nu_{1}-5\right) z_{1}^{2} \\
& +2 \phi_{11}^{2}(|\eta|)+\phi_{21}^{2}(|\eta|)+d_{2}(t) z_{2} z_{3}+z_{2}\left(d_{2}(t) \vartheta_{2}\right. \\
& \left.+z_{2} \widehat{\phi}_{2}\left(\bar{x}_{2}, \widehat{p}\right) \widehat{p}+z_{2} \bar{\phi}_{21}\left(\bar{x}_{2}, \widehat{p}\right)-\frac{\partial \vartheta_{1}}{\partial \widehat{p}} \tau_{2}\right)+\left(\frac{1}{\Upsilon} \tilde{p}\right. \\
& \left.+z_{2} \frac{\partial \vartheta_{1}}{\partial \widehat{p}}\right)\left(\tau_{2}-\dot{\hat{p}}\right) .
\end{aligned}
$$

For the term of $-z_{2}\left(\partial \vartheta_{1} / \partial \widehat{p}\right) \tau_{2}$, according to (33), it can be dealt with as follows:

$$
\begin{aligned}
& -z_{2} \frac{\partial \vartheta_{1}}{\partial \widehat{p}} \tau_{2}=-z_{2} \frac{\partial \vartheta_{1}}{\partial \widehat{p}} \Upsilon\left(z_{1}^{2} \widehat{\phi}_{1}\left(x_{1}\right)+z_{2}^{2} \widehat{\phi}_{2}\left(\bar{x}_{2}, \widehat{p}\right)\right) \\
& \leq z_{1}^{2}+\frac{1}{4} \Upsilon^{2} z_{2}^{2}\left(\frac{\partial \vartheta_{1}}{\partial \widehat{p}}\right)^{2} z_{1}^{2} \widehat{\phi}_{1}^{2}\left(x_{1}\right)+z_{2}^{2} \frac{1}{4} \Upsilon\left(1+z_{2}^{2}\right) \\
& \cdot \widehat{\phi}_{2}\left(\bar{x}_{2}, \widehat{p}\right)\left(1+\left(\frac{\partial \vartheta_{1}}{\partial \widehat{p}}\right)^{2}\right)=z_{1}^{2} \\
& +z_{2}^{2}\left(\frac{1}{4} \Upsilon^{2}\left(\frac{\partial \vartheta_{1}}{\partial \widehat{p}}\right)^{2} z_{1}^{2} \widehat{\phi}_{1}^{2}\left(x_{1}\right)\right. \\
& \left.+\frac{1}{4} \Upsilon\left(1+z_{2}^{2}\right) \widehat{\phi}_{2}\left(\bar{x}_{2}, \widehat{p}\right)\left(1+\left(\frac{\partial \vartheta_{1}}{\partial \widehat{p}}\right)^{2}\right)\right)
\end{aligned}
$$

Define the following smooth function: $\bar{\phi}_{22}\left(\bar{x}_{2}, \widehat{p}\right)=(1 /$ 4) $\Upsilon^{2} z_{1}^{2}\left(\partial \vartheta_{1} / \partial \widehat{p}\right)^{2} \widehat{\phi}_{1}^{2}\left(x_{1}\right)+(1 / 4) \Upsilon\left(1+z_{2}^{2}\right) \widehat{\phi}_{2}\left(\bar{x}_{2}, \widehat{p}\right)\left(1+\left(\partial \vartheta_{1} /\right.\right.$ $\left.\partial \widehat{p})^{2}\right)$, and we get

$$
-z_{2} \frac{\partial \vartheta_{1}}{\partial \widehat{p}} \tau_{2} \leq z_{1}^{2}+z_{2}^{2} \bar{\phi}_{22}\left(\bar{x}_{2}, \widehat{p}\right) .
$$

Denote $\bar{\phi}_{2}\left(\bar{x}_{2}, \widehat{p}\right)=\bar{\phi}_{21}\left(\bar{x}_{2}, \widehat{p}\right)+\bar{\phi}_{22}\left(\bar{x}_{2}, \widehat{p}\right)>0$, and a direct substitution leads to

$$
\begin{aligned}
\dot{V}_{2} \leq & -(1-\epsilon) \rho \circ \underline{\alpha}(|\eta|) \alpha_{0}(|\eta|)-\left(\nu_{1}-6\right) z_{1}^{2} \\
& +2 \phi_{11}^{2}(|\eta|)+\phi_{21}^{2}(|\eta|)+d_{2}(t) z_{2} z_{3} \\
& +z_{2}\left(d_{2}(t) \vartheta_{2}+z_{2} \widehat{\phi}_{2}\left(\bar{x}_{2}, \widehat{p}\right) \widehat{p}+z_{2} \bar{\phi}_{2}\left(\bar{x}_{2}, \widehat{p}\right)\right) \\
& +\left(\frac{1}{\Upsilon} \widetilde{p}+z_{2} \frac{\partial \vartheta_{1}}{\partial \hat{p}}\right)\left(\tau_{2}-\dot{\hat{p}}\right) .
\end{aligned}
$$

Take the virtual control

$$
\vartheta_{2}=-\frac{1}{\underline{d}_{2}}\left(\nu_{2}+\widehat{\phi}_{2}\left(\bar{x}_{2}, \widehat{p}\right) \hat{p}+\bar{\phi}_{2}\left(\bar{x}_{2}, \widehat{p}\right)\right) z_{2},
$$

which is such that

$$
\begin{aligned}
\dot{V}_{2} \leq & -(1-\epsilon) \rho \circ \underline{\alpha}(|\eta|) \alpha_{0}(|\eta|)-\left(\nu_{1}-6\right) z_{1}^{2}-\nu_{2} z_{2}^{2} \\
& +2 \phi_{11}^{2}(|\eta|)+\phi_{21}^{2}(|\eta|)+d_{2}(t) z_{2} z_{3} \\
& +\left(\frac{1}{\Upsilon} \tilde{p}+z_{2} \frac{\partial \vartheta_{1}}{\partial \hat{p}}\right)\left(\tau_{2}-\dot{\hat{p}}\right) .
\end{aligned}
$$

Step $i(3 \leq i \leq n)$. Assume that, in Step $i-1$, we have designed the virtual control $\vartheta_{j}(j=1, \ldots, i-1)$ and the tuning function $\tau_{j}(j=1, \ldots, i-1)$, such that the Lyapunov function

$$
V_{i-1}=\sum_{j=1}^{i-1} \frac{1}{2} z_{j}^{2}+\frac{1}{2 \Upsilon} \widetilde{p}^{2}
$$

satisfies

$$
\begin{aligned}
\dot{V}_{i-1} \leq & -(1-\epsilon) \rho \circ \underline{\alpha}(|\eta|) \alpha_{0}(|\eta|) \\
& -\left(\nu_{1}-6-\sum_{j=3}^{i-1}(2 j+1)\right) z_{1}^{2} \\
& -\left(\nu_{2}-7-\sum_{j=4}^{i-1}(2 j)\right) z_{2}^{2}-\cdots \\
& -\left(\nu_{i-2}-7\right) z_{i-2}^{2}-v_{i-1} z_{i-1}^{2} \\
& +\left(\frac{1}{\Upsilon} \tilde{p}+\sum_{j=2}^{i-1} z_{j} \frac{\partial \vartheta_{j-1}}{\partial \widehat{p}}\right)\left(\tau_{i-1}-\dot{\hat{p}}\right) \\
& +d_{i-1}(t) z_{i-1} z_{i}+\sum_{j=1}^{i-1}(i-j) \phi_{j 1}^{2}(|\eta|) .
\end{aligned}
$$

In what follows, it will be shown that the property (42) also holds in Step $i$.

Let $\vartheta_{i}$ be the virtual control and $z_{i+1}=x_{i+1}-\vartheta_{i}$. Consider the Lyapunov function

$$
V_{i}=V_{i-1}+\frac{1}{2} z_{i}^{2}
$$

To begin with, the dynamics of $z_{i}$ can be expressed as

$$
\begin{aligned}
\dot{z}_{i}= & d_{i}(t) \vartheta_{i}+g_{i}(t, \eta, x)-\sum_{j=1}^{i-1} \frac{\partial \vartheta_{i-1}}{\partial x_{j}} g_{j}(t, \eta, x) \\
& -\sum_{j=1}^{i-1} \frac{\partial \vartheta_{i-1}}{\partial x_{j}} d_{j}(t) x_{j+1}-\frac{\partial \vartheta_{i-1}}{\partial \hat{p}} \dot{\hat{p}}+d_{i}(t) z_{i+1} .
\end{aligned}
$$

For notational convenience, denote $\vartheta_{0}=0$. From $x_{k}=$ $z_{k}+\vartheta_{k-1}=z_{k}-\left(1 / \underline{d}_{k-1}\right)\left(\nu_{k-1}+\widehat{\phi}_{k-1}\left(x_{[k-1]}, \widehat{p}\right) \hat{p}+\right.$ 
$\left.\bar{\phi}_{i}\left(x_{[k-1]}, \widehat{p}\right)\right) z_{k-1}(k=1, \ldots, i-1)$, like the calculations in (26), it can be verified that there exist smooth functions $\widehat{\phi}_{i j}(\cdot)(j=1, \ldots, i)>0$, such that, for $j=1, \ldots, i-1$,

$$
\begin{aligned}
-z_{i} \frac{\partial \vartheta_{i-1}}{\partial x_{j}} g_{j}(t, \eta, x) \leq & \phi_{j 1}^{2}(|\eta|)+\sum_{k=1}^{j-1}\left(2 z_{k}^{2}\right)+z_{j}^{2} \\
& +z_{i}^{2} \widehat{\phi}_{i j}\left(\bar{x}_{j}, \widehat{p}\right) p^{*}, \\
z_{i} g_{i}(t, \eta, x) \leq & \phi_{i 1}^{2}(|\eta|) \\
& +\sum_{k=1}^{i-1}\left(2 z_{k}^{2}\right)+z_{i}^{2} \widehat{\phi}_{i i}\left(\bar{x}_{i}, \widehat{p}\right) p^{*} .
\end{aligned}
$$

Define $\widehat{\phi}_{i}\left(\bar{x}_{i}, \widehat{p}\right)=\sum_{j=1}^{i} \widehat{\phi}_{i j}\left(\bar{x}_{j}, \widehat{p}\right)$; from (45), it follows that

$$
\begin{aligned}
& z_{i}\left(g_{i}(t, \eta, x)-\sum_{j=1}^{i-1} \frac{\partial \vartheta_{i-1}}{\partial x_{j}} g_{j}(t, \eta, x)\right) \\
& \leq \sum_{j=1}^{i} \phi_{j 1}^{2}(|\eta|) \\
& \quad+\sum_{j=1}^{i-1}(1+2(i-j)) z_{j}^{2}+z_{i}^{2} \widehat{\phi}_{i}\left(\bar{x}_{i}, \hat{p}\right) p^{*} .
\end{aligned}
$$

Similar to (31) and (32), there exists a smooth function $\bar{\phi}_{i 1}\left(\bar{x}_{i}, \widehat{p}\right)$ such that

$$
\begin{aligned}
& z_{i}\left(d_{i-1}(t) z_{i-1}-\sum_{j=1}^{i-1} \frac{\partial \vartheta_{i-1}}{\partial x_{j}} d_{j}(t) x_{j+1}\right) \\
& \quad \leq z_{1}^{2}+\sum_{k=2}^{i-2}\left(2 z_{k}^{2}\right)+3 z_{i-1}^{2}+z_{i}^{2} \bar{\phi}_{i 1}\left(\bar{x}_{i}, \hat{p}\right) .
\end{aligned}
$$

Let

$$
\tau_{i}=\tau_{i-1}+\Upsilon z_{i}^{2} \widehat{\phi}_{i}\left(\bar{x}_{i}, \widehat{p}\right),
$$

and then, from (47) and (48), there holds

$$
\begin{aligned}
\dot{V}_{i} \leq & -(1-\epsilon) \rho \circ \underline{\alpha}(|\eta|) \alpha_{0}(|\eta|)-\left(v_{1}-6\right. \\
& \left.-\sum_{j=3}^{i-1}(2 j+1)-2 i\right) z_{1}^{2}-\left(\nu_{2}-7\right. \\
& \left.-\sum_{j=4}^{i-1}(2 j)-1-2(i-1)\right) z_{2}^{2}-\cdots-\left(v_{i-2}-7-7\right) \\
& \cdot z_{i-2}^{2}-\left(v_{i-1}-6\right) z_{i-1}^{2}+\sum_{j=1}^{i}(i-j+1) \phi_{j 1}^{2}(|\eta|) \\
& +d_{i}(t) z_{i} z_{i+1}+z_{i}\left(d_{i}(t) \vartheta_{i}+z_{i} \widehat{\phi}_{i}\left(\bar{x}_{i}, \widehat{p}\right) \widehat{p}\right. \\
& +z_{i} \bar{\phi}_{i 1}\left(\bar{x}_{i}, \widehat{p}\right)
\end{aligned}
$$

$$
\begin{aligned}
& \left.-\sum_{\mathrm{j}=2}^{i-1} z_{j} \frac{\partial \vartheta_{j-1}}{\partial \widehat{p}} \cdot \Upsilon z_{i} \widehat{\phi}_{i}\left(\bar{x}_{i}, \widehat{p}\right)-\frac{\partial \vartheta_{i-1}}{\partial \widehat{p}} \tau_{i}\right)+\left(\frac{1}{\Upsilon} \widetilde{p}\right. \\
& \left.+\sum_{j=2}^{i} z_{j} \frac{\partial \vartheta_{j-1}}{\partial \widehat{p}}\right)\left(\tau_{i}-\dot{\hat{p}}\right) .
\end{aligned}
$$

Remark 10. In (49), we subtract two terms $-\sum_{j=2}^{i-1} z_{j}\left(\partial \vartheta_{j-1} /\right.$ $\partial \widehat{p}) \cdot \Upsilon z_{i} \widehat{\phi}_{i}\left(\bar{x}_{i}, \widehat{p}\right)$ and $-\left(\partial \vartheta_{i-1} / \partial \widehat{p}\right) \tau_{i}$ in the brackets to generate the term $\left((1 / \Upsilon) \tilde{p}+\sum_{j=2}^{i} z_{j}\left(\partial \vartheta_{j-1} / \partial \widehat{p}\right)\right)\left(\tau_{i}-\dot{\hat{p}}\right)$.

However, from (49), it can be seen that, due to the unknown control coefficient $d_{i}(t)$, the terms $-\sum_{j=2}^{i-1} z_{j}\left(\partial \vartheta_{j-1} /\right.$ $\partial \widehat{p}) \cdot z_{i} \widehat{\phi}_{i}\left(\bar{x}_{i}, \widehat{p}\right)$ and $-\left(\partial \vartheta_{i-1} / \partial \widehat{p}\right) \tau_{i}$ could not be directly canceled by the coming virtual control $\vartheta_{i}$. We get around this burden by the following estimates:

$$
\begin{aligned}
& -z_{i} \sum_{j=2}^{i-1} z_{j} \frac{\partial \vartheta_{j-1}}{\partial \widehat{p}} \cdot \Upsilon z_{i} \widehat{\phi}_{i}\left(\bar{x}_{i}, \widehat{p}\right) \\
& \leq z_{i}^{2} \frac{1}{2}\left(1+\Upsilon^{2}\left(\sum_{j=2}^{i-1} z_{j} \frac{\partial \vartheta_{j-1}}{\partial \widehat{p}}\right)^{2} \widehat{\phi}_{i}^{2}\left(\bar{x}_{i}, \widehat{p}\right)\right), \\
& -z_{i} \frac{\partial \vartheta_{i-1}}{\partial \widehat{p}} \tau_{i}=-z_{i} \frac{\partial \vartheta_{i-1}}{\partial \widehat{p}} \Upsilon\left(z_{1}^{2} \widehat{\phi}_{1}+\cdots+z_{i}^{2} \widehat{\phi}_{i}\right) \\
& \leq \sum_{j=1}^{i-1} z_{j}^{2}+z_{i}^{2} \frac{1}{4} \Upsilon^{2}\left(\frac{\partial \vartheta_{i-1}}{\partial \widehat{p}}\right)^{2} \sum_{j=1}^{i} z_{j}^{2} \widehat{\phi}_{j}^{2}\left(\bar{x}_{j}, \widehat{p}\right) .
\end{aligned}
$$

Let $\bar{\phi}_{i}\left(\bar{x}_{i}, \widehat{p}\right)=(1 / 4) \Upsilon^{2}\left(\partial \vartheta_{i-1} / \partial \widehat{p}\right)^{2} \sum_{j=1}^{i} z_{j}^{2} \widehat{\phi}_{j}^{2}\left(\bar{x}_{j}, \widehat{p}\right)+(1 /$ $2)\left(1+\Upsilon^{2}\left(\sum_{j=2}^{i-1} z_{j}\left(\partial \vartheta_{j-1} / \partial \widehat{p}\right)\right)^{2} \widehat{\phi}_{i}^{2}\left(\bar{x}_{i}, \widehat{p}\right)\right)+\bar{\phi}_{i 1}\left(\bar{x}_{i}, \widehat{p}\right)>0$, and then we get

$$
\begin{gathered}
z_{i}\left(z_{i} \bar{\phi}_{i 1}\left(\bar{x}_{i}, \widehat{p}\right)-\sum_{j=2}^{i-1} z_{j} \frac{\partial \vartheta_{j-1}}{\partial \widehat{p}} \cdot z_{i} \widehat{\phi}_{i}\left(\bar{x}_{i}, \widehat{p}\right)\right. \\
\left.-\frac{\partial \vartheta_{i-1}}{\partial \widehat{p}} \tau_{i}\right) \leq \sum_{j=1}^{i-1} z_{j}^{2}+z_{i}^{2} \bar{\phi}_{i}\left(\bar{x}_{i}, \widehat{p}\right) .
\end{gathered}
$$

By substituting (51) into (49), it follows that

$$
\begin{aligned}
\dot{V}_{i} \leq & -(1-\epsilon) \rho \circ \underline{\alpha}(|\eta|) \alpha_{0}(|\eta|) \\
& -\left(v_{1}-6-\sum_{j=3}^{i}(2 j+1)\right) z_{1}^{2} \\
& -\left(v_{2}-7-\sum_{j=4}^{i}(2 j)\right) z_{2}^{2}-\cdots \\
& -\left(v_{i-2}-7-8\right) z_{i-2}^{2}-\left(v_{i-1}-7\right) z_{i-1}^{2} \\
& +z_{i}\left(d_{i}(t) \vartheta_{i}+z_{i} \widehat{\phi}_{i}\left(\bar{x}_{i}, \hat{p}\right) \hat{p}+z_{i} \bar{\phi}_{i}\left(\bar{x}_{i}, \widehat{p}\right)\right)
\end{aligned}
$$




$$
\begin{aligned}
& +\left(\frac{1}{\Upsilon} \tilde{p}+\sum_{j=2}^{i} z_{j} \frac{\partial \vartheta_{j-1}}{\partial \widehat{p}}\right)\left(\tau_{i}-\dot{\hat{p}}\right) \\
& +\sum_{j=1}^{i}(i-j+1) \phi_{j 1}^{2}(|\eta|)+d_{i}(t) z_{i} z_{i+1}
\end{aligned}
$$

Take the virtual control

$$
\vartheta_{i}=-\frac{1}{\underline{d}_{i}}\left(v_{i}+\widehat{\phi}_{i}\left(\bar{x}_{i}, \widehat{p}\right) \widehat{p}+\bar{\phi}_{i}\left(\bar{x}_{i}, \widehat{p}\right)\right) z_{i},
$$

and then the following holds:

$$
\begin{aligned}
\dot{V}_{i} \leq & -(1-\epsilon) \rho \circ \underline{\alpha}(|\eta|) \alpha_{0}(|\eta|) \\
& -\left(v_{1}-6-\sum_{j=3}^{i}(2 j+1)\right) z_{1}^{2} \\
& -\left(v_{2}-7-\sum_{j=4}^{i}(2 j)\right) z_{2}^{2}-\cdots-\left(v_{i-1}-7\right) z_{i-1}^{2} \\
& -v_{i} z_{i}^{2}+\sum_{j=1}^{i}(i-j+1) \phi_{j 1}^{2}(|\eta|) \\
& +\left(\frac{1}{\Upsilon} \widetilde{p}+\sum_{j=2}^{i} z_{j} \frac{\partial \vartheta_{j-1}}{\partial \widehat{p}}\right)\left(\tau_{i}-\dot{\hat{p}}\right)+d_{i}(t) z_{i} z_{i+1} .
\end{aligned}
$$

In particular, when $i=n$, the actual control $u$ appears, and we choose the controller $u$ and updating law $\tau_{n}$ for $\widehat{p}(t)$ as follows:

$$
\begin{aligned}
& u=-\frac{1}{\underline{d}_{n}}\left(v_{n}+\widehat{\phi}_{n}(x, \widehat{p}) \widehat{p}+\bar{\phi}_{n}(x, \widehat{p})\right) z_{n}, \\
& \dot{\hat{p}}=\tau_{n}=\sum_{j=1}^{n} \Upsilon z_{j}^{2} \widehat{\phi}_{j}\left(\bar{x}_{j}, \widehat{p}\right),
\end{aligned}
$$

such that the Lyapunov function

$$
V_{n}=\sum_{j=1}^{n} \frac{1}{2} z_{j}^{2}+\frac{1}{2 \Upsilon} \widetilde{p}^{2}
$$

satisfies

$$
\begin{aligned}
\dot{V}_{n} \leq & -(1-\epsilon) \rho \circ \underline{\alpha}(|\eta|) \alpha_{0}(|\eta|) \\
& -\left(\nu_{1}-6-\sum_{j=3}^{n}(2 j+1)\right) z_{1}^{2} \\
& -\left(v_{2}-7-\sum_{j=4}^{n}(2 j)\right) z_{2}^{2}-\cdots-\left(v_{n-1}-7\right) z_{n-1}^{2} \\
& -v_{n} z_{n}^{2}+\sum_{j=1}^{n}(n-j+1) \phi_{j 1}^{2}(|\eta|) .
\end{aligned}
$$

This completes the controller design procedure.

\section{Main Results}

After the above controller design procedure, we are now ready to state the main results.

Theorem 11. Suppose the investigated system (1) satisfies Assumptions 1, 3, and 4 together with the local conditions

$$
\phi_{i 1}^{2}(s)=O\left(\alpha_{0}(s)\right), \quad i=1, \ldots, n .
$$

Then all the signals of the closed-loop system (1) with the controller (55) and updating law (56) are bounded on $[0,+\infty)$. Specifically, the following convergent property holds:

$$
\lim _{t \rightarrow \infty}(|\eta(t)|+|x(t)|+|u(t)|)=0
$$

Proof. From the local conditions (59), one can choose the smooth function $\rho(\cdot)$ such that

$$
\sum_{j=1}^{n}(n-j+1) \phi_{j 1}^{2}(|\eta|) \leq \frac{1-\epsilon}{2} \rho \circ \underline{\alpha}(|\eta|) \alpha_{0}(|\eta|) .
$$

One can choose positive constants $v_{j}(j=1, \ldots, n)$ satisfying

$$
\begin{gathered}
v_{1}-6-\sum_{j=3}^{n}(2 j+1) \geq 1, \\
v_{2}-7-\sum_{j=4}^{n}(2 j) \geq 1, \\
\vdots \\
v_{n-1}-7 \geq 1, \\
v_{n} \geq 1 .
\end{gathered}
$$

Then, from (58), (61), and (62), it follows that

$$
\dot{V}_{n} \leq-\frac{1-\epsilon}{2} \rho \circ \underline{\alpha}(|\eta|) \alpha_{0}(|\eta|)-\sum_{i=1}^{n} z_{i}^{2} .
$$

Now, assume that the maximal interval of existence of the solution of the closed-loop system starting from any given initial conditions is $\left[0, t_{f}\right)$ for some $t_{f}>0$. In view of $0<$ $\epsilon<1$, from (63), it can be concluded that $V_{n}$ and hence the variables $\left(\eta(t), z_{1}(t), \ldots, z_{n}(t), \widetilde{p}(t)\right)$ are bounded on $\left[0, t_{f}\right)$. In terms of $\widetilde{p}(t)=p^{*}-\widehat{p}(t)$, we obtain the boundedness of $\widehat{p}(t)$. Considering (53), it can be derived that $\vartheta_{i}(i=1, \ldots, n)$ are bounded. In view of $z_{i}=x_{i}-\vartheta_{i-1}\left(\vartheta_{0}=0\right)$, we further obtain that the states $x_{i}(i=1, \ldots, n)$ are bounded on $\left[0, t_{f}\right)$.

So far all the closed-loop system signals are bounded on $\left[0, t_{f}\right)$. This guarantees that the finite time escape will not happen. Therefore, it is natural that $t_{f}$ can be maximized to $+\infty$ by means of Theorem 3.3 in [21]. Next we will prove the convergence property of (60).

Again, according to (63), considering $\underline{\alpha}$ and $\alpha_{0}$ are $K_{\infty}$-functions, a direct application of LaSalle's invariance 
principle in [21] guarantees the convergence property of $\left(\eta(t), z_{1}(t), \ldots, z_{n}(t)\right)$; that is,

$$
\begin{aligned}
& \lim _{t \rightarrow \infty} \eta(t)=0, \\
& \lim _{t \rightarrow \infty} z_{i}(t)=0 \quad(i=1, \ldots, n) .
\end{aligned}
$$

As a consequence, from (53) and (55), the following holds:

$$
\lim _{t \rightarrow \infty} \vartheta_{i}(t)=0 \quad(i=1, \ldots, n) .
$$

Particularly,

$$
\lim _{t \rightarrow \infty} u(t)=0
$$

In terms of (64), (65), and $x_{i}=z_{i}+\vartheta_{i-1}(i=1, \ldots, n)$, we can obtain

$$
\lim _{t \rightarrow \infty} x_{i}(t)=0 \quad(i=1, \ldots, n) .
$$

This completes the proof.

It is noted that, under some stronger conditions, the designed control law can be a linear controller. In fact, we have the following statement.

Theorem 12. Suppose that the conditions for Theorem 11 are satisfied with $\delta_{0}$ known a priori and the following additional assumptions hold:

(i) The uncertain functions $\phi_{i 1}(\cdot)(i=1, \ldots, n)$ satisfy

$$
\limsup _{s \rightarrow+\infty} \frac{\phi_{i 1}^{2}(s)}{\alpha_{0}(s)}<+\infty \text {. }
$$

(ii) There exist known constants $p_{i j}>0(i=1, \ldots, n ; j=$ $1,2)$, such that

$$
\begin{array}{r}
\left|g_{i}(t, \eta, x)\right| \leq p_{i 1} \phi_{i 1}(|\eta|)+p_{i 2}\left(\left|x_{1}\right|+\cdots+\left|x_{i}\right|\right), \\
i=1, \ldots, n .
\end{array}
$$

(iii) $\gamma_{0}(|s|)=k s^{2}$ where $k$ is a positive constant.

Then, the proposed design method can result in a linear control law

$$
\begin{aligned}
u & =-\frac{1}{\underline{d}_{n}} v_{n} z_{n} \\
& =-\frac{1}{\underline{d}_{n}} v_{n}\left(x_{n}+v_{n-1}\left(x_{n-1}+\cdots+v_{1} x_{1}\right)\right),
\end{aligned}
$$

where $v_{i}(i=1, \ldots, n)$ are some sufficiently large positive constants.

Proof. Under the above hypotheses (i)-(iii), it is known that the constant $p^{*}$ is known, and hence the estimation $\widehat{p}$ for $p^{*}$ is no longer needed. Moreover, since conditions (59) and (68) are satisfied, the function $\rho(\cdot)$ in (7) can be chosen as a constant $\rho>0$. For $i=1, \ldots, n$, we consider the following function:

$$
\bar{V}_{0 i}(\eta)=\int_{0}^{V_{0}(\eta)} \rho_{i} d s
$$

where $\rho_{i}(i=1, \ldots, n)$ are design constants. In view of (3), we can get

$$
\begin{aligned}
\dot{\bar{V}}_{0 i}(\eta) \leq & -\rho_{i} \alpha_{0}(|\eta|)+\rho_{i} \delta_{0} \gamma_{0}(|y|) \\
= & -\phi_{i 1}^{2}(|\eta|)+\widetilde{\rho}_{i} \delta_{0} \gamma_{0}(|y|)+\phi_{i 1}^{2}(|\eta|) \\
& -\rho_{i} \alpha_{0}(|\eta|)-\tilde{\rho}_{i} \delta_{0} \gamma_{0}(|y|)+\rho_{i} \delta_{0} \gamma_{0}(|y|)
\end{aligned}
$$

with some positive constants $\tilde{\rho}_{i}(i=1, \ldots, n)$. We will prove that if the constants $\rho_{i}$ and $\tilde{\rho}_{i}$ are chosen suitably, the following inequality holds:

$$
\begin{array}{r}
\phi_{i 1}^{2}(|\eta|)-\rho_{i} \alpha_{0}(|\eta|)-\widetilde{\rho}_{i} \delta_{0} \gamma_{0}(|y|)+\rho_{i} \delta_{0} \gamma_{0}(|y|) \leq 0, \\
i=1, \ldots, n .
\end{array}
$$

In fact, because of $\phi_{i 1}^{2}(s)=O\left(\alpha_{0}(s)\right)$, there exist positive constants $s_{i}>0, c_{i}>0$ satisfying $\phi_{i 1}^{2}(s) \leq c_{i} \alpha_{0}(s)$, for $s \in\left[0, s_{i}\right]$. Take $\rho_{i} \geq c_{i}+1, \widetilde{\rho}_{i}=\rho_{i}+1$, and then $\phi_{i 1}^{2}(s)-\rho_{i} \alpha_{0}(s) \leq 0$ and $\left(\rho_{i}-\tilde{\rho}_{i}\right) \delta_{0} \gamma_{0}(|y|) \leq 0$. In view of $\limsup _{s \rightarrow \infty}\left(\phi_{i 1}^{2}(s) / \alpha_{0}(s)\right)<\infty$, there exist positive constants $s_{i}^{\prime}>0, c_{i}^{\prime}>0$ satisfying $\phi_{i 1}^{2}(s) \leq c_{i}^{\prime} \alpha_{0}(s)$ if $s \in\left[s_{i}^{\prime}, \infty\right]$. Similarly, if we take $\rho_{i} \geq c_{i}^{\prime}+1$ and $\widetilde{\rho}_{i}=\rho_{i}+1$, then $\phi_{i 1}^{2}(s)-\rho_{i} \alpha_{0}(s) \leq 0,\left(\rho_{i}-\widetilde{\rho}_{i}\right) \delta_{0} \gamma_{0}(|y|) \leq 0$. In the finite closed interval $\left[s_{i}, s_{i}^{\prime}\right]$, let $\alpha_{0}(\underline{s})$ be the minimum value of $\alpha_{0}(s)$ and let $\phi_{i 1}^{2}(\bar{s})$ be the maximum value of $\phi_{i 1}^{2}(s)$, respectively, and if we take $\rho_{i} \geq \phi_{i 1}^{2}(\bar{s}) / \alpha_{0}(\underline{s}), \widetilde{\rho}_{i}=\rho_{i}+1$, then $\phi_{i 1}^{2}(s)-\rho_{i} \alpha_{0}(s) \leq 0$, $\left(\rho_{i}-\widetilde{\rho}_{i}\right) \delta_{0} \gamma_{0}(|y|) \leq 0$. According to the previous analysis, we choose $\rho_{i}=c_{i}+1+c_{i}^{\prime}+1+\phi_{i 1}^{2}(\bar{s}) / \alpha_{0}(\underline{s})$ and $\tilde{\rho}_{i}=\rho_{i}+1$, and then (72) holds. Therefore we get

$$
\dot{\bar{V}}_{0 i}(\eta) \leq-\phi_{i 1}^{2}(|\eta|)+\widetilde{\rho}_{i} \delta_{0} \gamma_{0}(|y|), \quad i=1, \ldots, n .
$$

In view of $\gamma_{0}(s)=k s^{2}$, a direct substitution in (74) results in

$$
\dot{\bar{V}}_{0 i}(\eta) \leq-\phi_{i 1}^{2}(|\eta|)+\widetilde{\rho}_{i} \delta_{0} k y^{2}, \quad i=1, \ldots, n .
$$

To deal with the unmeasured dynamics $\eta$ in this case, we can choose the candidate Lyapunov function as follows:

$$
V_{n}=\sum_{i=1}^{n} \frac{1}{2} z_{i}^{2}+\sum_{i=1}^{n}(n-i+2) \bar{V}_{0 i}(\eta) .
$$

Consequently, a modified version of the design procedure in Section 3 leads to the linear control law

$$
\begin{aligned}
u & =-\frac{1}{\underline{d}_{n}} v_{n} z_{n} \\
& =-\frac{1}{\underline{d}_{n}} v_{n}\left(x_{n}+v_{n-1}\left(x_{n-1}+\cdots+v_{1} x_{1}\right)\right)
\end{aligned}
$$

with some sufficiently large positive constants $v_{i}(i=$ $1, \ldots, n)$. 


\section{Simulation Example}

In this section, we provide a simulation example to illustrate the proposed method in the paper. Consider the following nonlinear systems:

$$
\begin{aligned}
\dot{\eta} & =-\eta+\delta(t) y^{2}, \\
\dot{x}_{1} & =d_{1}(t) x_{2}+\theta_{1} x_{1}+\theta_{2} \frac{x_{1} x_{2}}{1+x_{2}^{2}}, \\
\dot{x}_{2} & =d_{2}(t) u+\theta_{3} \sin \left(t x_{1} x_{2}\right) \eta, \\
y & =x_{1}
\end{aligned}
$$

with $q(t, \eta, y)=-\eta+\delta(t) y^{2}, \delta(t)=1+e^{-t}, g_{1}(t, \eta, x)=$ $\theta_{1} x_{1}+\theta_{2}\left(x_{1} x_{2} /\left(1+x_{2}^{2}\right)\right)$, and $g_{2}(t, \eta, x)=\theta_{3} \sin \left(t x_{1} x_{2}\right) \eta$. The inverse system $\dot{\eta}=-\eta+\delta(t) y^{2}$ is ISS, and $V_{0}(\eta)=(1 / 2) \eta^{2}$ is a candidate ISS-Lyapunov function with the supply pair $\alpha_{0}(|\eta|)=(1 / 2) \eta^{2}, \delta(t) \gamma_{0}\left(\left|x_{1}\right|\right)=\left(1+e^{-t}\right)(1 / 4) x_{1}^{4}$.

Next, we use the proposed algorithm in Section 3 to design the partial-state feedback controller.

Step 1. We consider the function $V_{1}=(1 / 2) z_{1}^{2}+(1 / 2) \eta^{2}$, whose time derivative satisfies

$$
\begin{aligned}
\dot{V}_{1}= & z_{1}\left(d_{1}(t) x_{2}+\theta_{1} x_{1}+\theta_{2} \frac{x_{1} x_{2}}{1+x_{2}^{2}}\right) \\
& +\eta\left(-\eta+\delta(t) y^{2}\right) .
\end{aligned}
$$

Like the calculations in (14), we have

$$
\begin{aligned}
z_{1} \theta_{1} x_{1} & \leq\left|\theta_{1}\right| z_{1}^{2}, \\
z_{1} \theta_{2} \frac{x_{1} x_{2}}{1+x_{2}^{2}} & \leq \frac{1}{2}\left|\theta_{2}\right| z_{1}^{2}, \\
\eta \delta(t) y^{2} & \leq \frac{1}{4} \eta^{2}+\delta^{2}(t) z_{1}^{4} \leq \frac{1}{4} \eta^{2}+\delta_{0} z_{1}^{4},
\end{aligned}
$$

where $\delta_{0}>0$ satisfying $\delta^{2}(t) \leq \delta_{0}$.

Define $p^{*}=\max \left\{\delta_{0},\left|\theta_{i}\right|, \theta_{i}^{2} \mid i=1,2,3\right\}$, and we get

$$
\begin{aligned}
& z_{1} \theta_{1} x_{1}+z_{1} \theta_{2} \frac{x_{1} x_{2}}{1+x_{2}^{2}}+\eta \delta(t) y^{2} \\
& \quad \leq \frac{1}{4} \eta^{2}+p^{*} z_{1}^{2}\left(1+\frac{1}{2}+z_{1}^{2}\right) \leq \frac{1}{4} \eta^{2}+z_{1}^{2} \widehat{\phi}_{1}\left(x_{1}\right) p^{*}
\end{aligned}
$$

with a new smooth function $\widehat{\phi}_{1}\left(x_{1}\right) \geq 1+1 / 2+z_{1}^{2}>0$.

Similar to (17), we augment $V_{1}$ as follows:

$$
\bar{V}_{1}=V_{1}+\frac{1}{2 \Upsilon} \widetilde{p}^{2}
$$

where $\Upsilon>0$ is the design parameter. In view of (79) and $p^{*}=\widehat{p}+\widetilde{p}$, a direct substitution leads to

$$
\begin{aligned}
\dot{\bar{V}}_{1} \leq & -\left(1-\frac{1}{4}\right) \eta^{2}+d_{1}(t) z_{1} \vartheta_{1}+d_{1}(t) z_{1} z_{2} \\
& +z_{1}^{2} \widehat{\phi}_{1}\left(x_{1}\right) \hat{p}+\frac{1}{\Upsilon} \widetilde{p}\left(\Upsilon \widehat{\phi}_{1}\left(x_{1}\right) z_{1}^{2}-\dot{\hat{p}}\right) .
\end{aligned}
$$

We take the virtual control and the tuning function

$$
\begin{aligned}
\vartheta_{1} & =-\frac{1}{\underline{d}_{1}}\left(\nu_{1}+\widehat{\phi}_{1}\left(x_{1}\right) \hat{p}\right) z_{1}, \quad \nu_{1}>0, \\
\tau_{1} & =\Upsilon \widehat{\phi}_{1}\left(x_{1}\right) z_{1}^{2},
\end{aligned}
$$

and then we get

$$
\begin{aligned}
\dot{\bar{V}}_{1} \leq & -\left(1-\frac{1}{4}\right) \eta^{2}-v_{1} z_{1}^{2}+d_{1}(t) z_{1} z_{2} \\
& +\frac{1}{\Upsilon} \widetilde{p}\left(\tau_{1}-\dot{\hat{p}}\right) .
\end{aligned}
$$

Step 2. To find the actual control law $u$, we consider the Lyapunov function

$$
V_{2}=\bar{V}_{1}+\frac{1}{2} z_{2}^{2}
$$

In view of (78) and (85), we have

$$
\begin{aligned}
\dot{V}_{2} & \leq-\left(1-\frac{1}{4}\right) \eta^{2}-v_{1} z_{1}^{2}+d_{1}(t) z_{1} z_{2}+\frac{1}{\Upsilon} \widetilde{p}\left(\tau_{1}\right. \\
& -\dot{\hat{p}})+z_{2}\left(d_{2}(t) u+\theta_{3} \sin \left(t x_{1} x_{2}\right) \eta\right. \\
& \left.-\frac{\partial \vartheta_{1}}{\partial x_{1}}\left(d_{1}(t) x_{2}+\theta_{1} x_{1}+\theta_{2} \frac{x_{1} x_{2}}{1+x_{2}^{2}}\right)-\frac{\partial \vartheta_{1}}{\partial \widehat{p}} \dot{\hat{p}}\right) .
\end{aligned}
$$

As in (80), we have

$$
\begin{aligned}
& d_{1}(t) z_{1} z_{2} \leq z_{1}^{2}+\frac{\bar{d}_{1}^{2}}{4} z_{2}^{2}, \\
& z_{2} \theta_{3} \sin \left(t x_{1} x_{2}\right) \eta \leq\left|z_{2} \theta_{3} \sin \left(t x_{1} x_{2}\right) \eta\right| \\
& \leq \frac{1}{4} \eta^{2}+\theta_{3}^{2} z_{2}^{2}, \\
& -z_{2} \frac{\partial \vartheta_{1}}{\partial x_{1}} \theta_{1} x_{1} \leq z_{1}^{2}+\frac{1}{4} \theta_{1}^{2} z_{2}^{2}\left(\frac{\partial \vartheta_{1}}{\partial x_{1}}\right)^{2}, \\
& -z_{2} \frac{\partial \vartheta_{1}}{\partial x_{1}} \theta_{2} \frac{x_{1} x_{2}}{1+x_{2}^{2}} \leq z_{1}^{2}+\frac{1}{4} \theta_{2}^{2} z_{2}^{2}\left(\frac{\partial \vartheta_{1}}{\partial x_{1}}\right)^{2} \frac{1}{4}, \\
& -z_{2} \frac{\partial \vartheta_{1}}{\partial x_{1}} d_{1}(t) x_{2} \\
& \leq z_{2}^{2} \frac{\bar{d}_{1}}{2}\left(1+\left(\frac{\partial \vartheta_{1}}{\partial x_{1}}\right)^{2}\right)+z_{1}^{2} \\
& \quad+\frac{1}{4} z_{2}^{2}\left(\frac{\partial \vartheta_{1}}{\partial x_{1}}\right)^{2} \frac{\bar{d}_{1}^{2}}{\underline{d}_{1}^{2}}\left(v_{1}+\widehat{\phi}_{1}\left(x_{1}\right) \widehat{p}\right)^{2} .
\end{aligned}
$$

Consequently, in view of the definition of $p^{*}$, the following holds:

$$
\begin{aligned}
& z_{2} \theta_{3} \sin \left(t x_{1} x_{2}\right) \eta-z_{2} \frac{\partial \vartheta_{1}}{\partial x_{1}} \theta_{1} x_{1}-z_{2} \frac{\partial \vartheta_{1}}{\partial x_{1}} \theta_{2} \frac{x_{1} x_{2}}{1+x_{2}^{2}} \\
& \leq \frac{1}{4} \eta^{2}+2 z_{1}^{2}+z_{2}^{2} \widehat{\phi}_{2}\left(x_{1}, \widehat{p}\right) p^{*},
\end{aligned}
$$



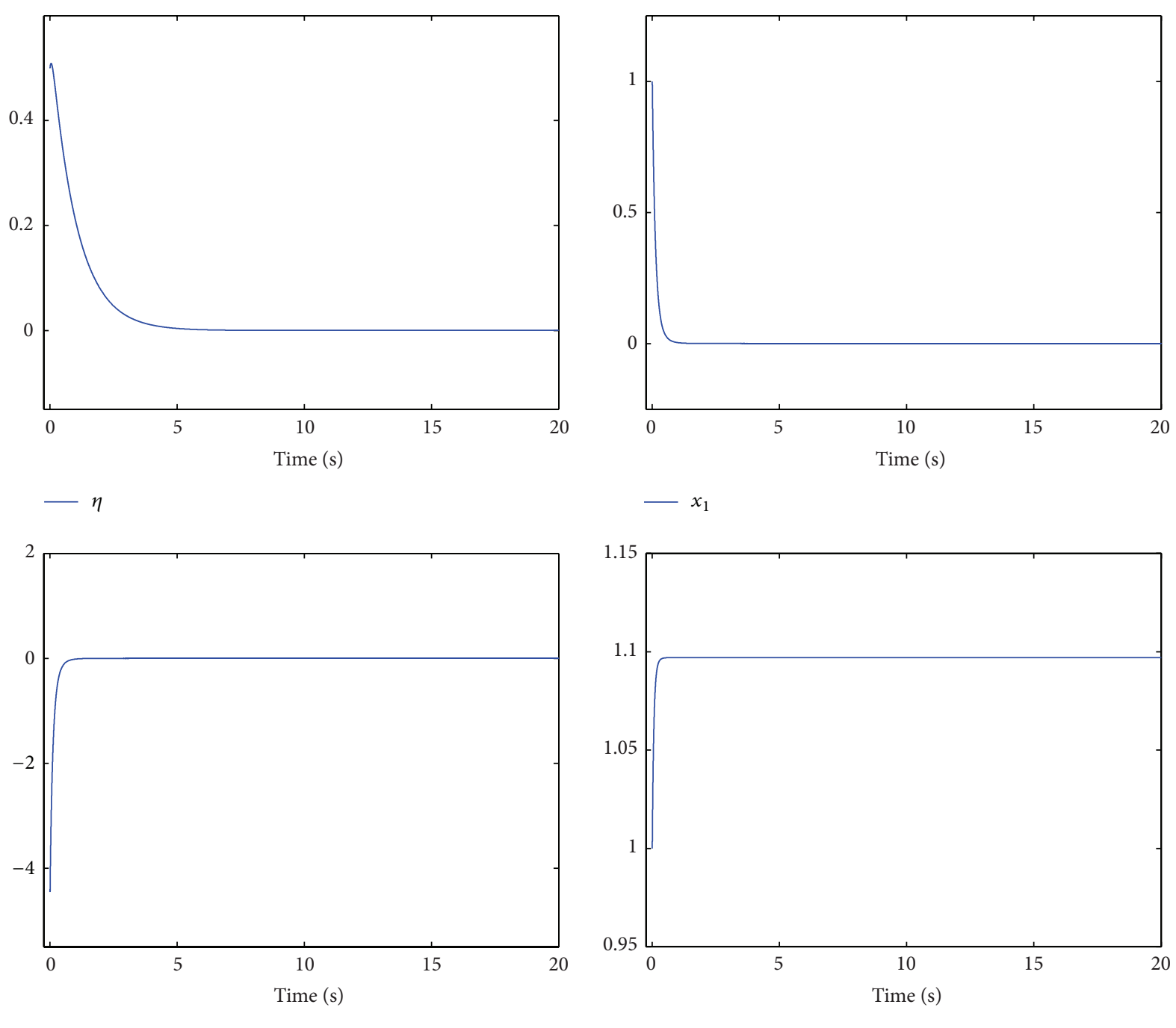

Figure 1: The response of the closed-loop system.

with $\widehat{\phi}_{2}\left(x_{1}, \widehat{p}\right)=1+(1 / 4)\left(\partial \vartheta_{1} / \partial x_{1}\right)^{2}+(1 / 4)\left(\partial \vartheta_{1} / \partial x_{1}\right)^{2}(1 / 4)$. Define $\bar{\phi}_{21}\left(x_{1}, \widehat{p}\right)=\bar{d}_{1}^{2} / 4+\left(\bar{d}_{1} / 2\right)\left(1+\left(\partial \vartheta_{1} / \partial x_{1}\right)^{2}\right)+(1 / 4)\left(\bar{d}_{1}^{2} /\right.$ $\left.\underline{d}_{1}^{2}\right)\left(\partial \vartheta_{1} / \partial x_{1}\right)^{2}\left(\nu_{1}+\widehat{\phi}_{1}\left(x_{1}\right) \hat{p}\right)^{2}$, and one gets

$$
z_{2}\left(d_{1}(t) z_{1}-\frac{\partial \vartheta_{1}}{\partial x_{1}} d_{1}(t) x_{2}\right) \leq 2 z_{1}^{2}+z_{2}^{2} \bar{\phi}_{21}\left(x_{1}, \widehat{p}\right) .
$$

As a result, with $\tau_{2}=\tau_{1}+\Upsilon z_{2}^{2} \widehat{\phi}_{2}\left(x_{1}, \widehat{p}\right)$, we have

$$
\begin{aligned}
\dot{V}_{2} \leq & -\left(1-\frac{2}{4}\right) \eta^{2}-\left(v_{1}-4\right) z_{1}^{2}+z_{2}^{2} \widehat{\phi}_{2}\left(x_{1}, \widehat{p}\right) \widehat{p} \\
& +z_{2}^{2} \bar{\phi}_{21}\left(x_{1}, \widehat{p}\right)+\left(\frac{1}{\Upsilon} \widetilde{p}+z_{2} \frac{\partial \vartheta_{1}}{\partial \widehat{p}}\right)\left(\tau_{2}-\dot{\hat{p}}\right) \\
& +z_{2} d_{2}(t) u-z_{2} \frac{\partial \vartheta_{1}}{\partial \widehat{p}} \tau_{2} .
\end{aligned}
$$

For the term of $-z_{2}\left(\partial \vartheta_{1} / \partial \widehat{p}\right) \tau_{2}$, according to (36), it can be verified that

$$
\begin{array}{r}
-z_{2} \frac{\partial \vartheta_{1}}{\partial \widehat{p}} \tau_{2} \leq z_{1}^{2}+z_{2}^{2}\left(\frac{1}{4} \Upsilon^{2}\left(\frac{\partial \vartheta_{1}}{\partial \widehat{p}}\right)^{2} z_{1}^{2} \widehat{\phi}_{1}^{2}\left(x_{1}\right)\right. \\
\left.+\frac{1}{4} \Upsilon\left(1+z_{2}^{2}\right) \widehat{\phi}_{2}\left(x_{1}, \widehat{p}\right)\left(1+\left(\frac{\partial \vartheta_{1}}{\partial \widehat{p}}\right)^{2}\right)\right) .
\end{array}
$$

Define $\bar{\phi}_{22}\left(\bar{x}_{2}, \widehat{p}\right)=(1 / 4) \Upsilon^{2} z_{1}^{2}\left(\partial \vartheta_{1} / \partial \widehat{p}\right)^{2} \widehat{\phi}_{1}^{2}\left(x_{1}\right)+(1 / 4) \Upsilon(1+$ $\left.z_{2}^{2}\right) \widehat{\phi}_{2}\left(x_{1}, \widehat{p}\right)\left(1+\left(\partial \vartheta_{1} / \partial \widehat{p}\right)^{2}\right)$, and we get

$$
-z_{2} \frac{\partial \vartheta_{1}}{\partial \widehat{p}} \tau_{2} \leq z_{1}^{2}+z_{2}^{2} \bar{\phi}_{22}\left(\bar{x}_{2}, \widehat{p}\right)
$$

Denote $\bar{\phi}_{2}\left(\bar{x}_{2}, \widehat{p}\right)=\bar{\phi}_{21}\left(x_{1}, \widehat{p}\right)+\bar{\phi}_{22}\left(\bar{x}_{2}, \widehat{p}\right)>0$, and a direct substitution leads to 


$$
\begin{aligned}
\dot{V}_{2} \leq & -\left(1-\frac{2}{4}\right) \eta^{2}-\left(\nu_{1}-5\right) z_{1}^{2} \\
& +z_{2}\left(d_{2}(t) u+z_{2} \widehat{\phi}_{2}\left(\bar{x}_{2}, \widehat{p}\right) \widehat{p}+z_{2} \bar{\phi}_{2}\left(\bar{x}_{2}, \widehat{p}\right)\right) \\
& +\left(\frac{1}{\Upsilon} \widetilde{p}+z_{2} \frac{\partial \vartheta_{1}}{\partial \widehat{p}}\right)\left(\tau_{2}-\dot{\hat{p}}\right) .
\end{aligned}
$$

As in Step 1, we design the following partial-state feedback controller:

$$
u=-\frac{1}{\underline{d}_{2}}\left(v_{2}+\widehat{\phi}_{2}\left(\bar{x}_{2}, \widehat{p}\right) \widehat{p}+\bar{\phi}_{2}\left(\bar{x}_{2}, \widehat{p}\right)\right) z_{2}
$$

with the updating law for unknown parameter $p^{*}$

$$
\dot{\hat{p}}=\Upsilon \widehat{\phi}_{1}\left(x_{1}\right) z_{1}^{2}+\Upsilon \widehat{\phi}_{2}\left(x_{1}, x_{2}, \widehat{p}\right) z_{2}^{2},
$$

which is such that

$$
\dot{V}_{2} \leq-\frac{1}{2} \eta^{2}-\left(v_{1}-5\right) z_{1}^{2}-v_{2} z_{2}^{2} .
$$

The Lyapunov function $V_{2}$ can be made $\dot{V}_{2} \leq 0$ by choosing $v_{1}>5, v_{2}>0$, and the stability analysis can be done in the similar way to Theorem 11. The simulation plots shown in Figures 1 and 2 are performed by MATLAB with the following parameters: $d_{1}(t)=2-\sin (t), d_{2}(t)=2+\sin (t)$, $\underline{d}_{1}=1, \bar{d}_{1}=3, \underline{d}_{2}=1, \bar{d}_{2}=3, v_{1}=6, v_{2}=1, \Upsilon=1$, the derived functions: $\phi_{11}(|\eta|)=0, \phi_{21}(|\eta|)=|\eta|, \phi_{12}\left(x_{1}\right)=$ $\left|x_{1}\right|+(1 / 2)\left|x_{1}\right|, \phi_{22}\left(x_{1}, x_{2}\right)=0, \widehat{\phi}_{1}\left(x_{1}\right)=1+1 / 2+x_{1}^{2}$, $\widehat{\phi}_{2}\left(x_{1}, x_{2}, \widehat{p}\right)=2+(3 / 2)\left(\partial \vartheta_{1} / \partial x_{1}\right)^{2}\left(v_{1}+\widehat{p}\right)^{2}$, and the initial conditions: $\eta(0)=0.5, x_{1}(0)=1, z_{2}(0)=0.1, \widehat{p}(0)=1$.

According to our results reported in Theorem 11, the states $\left(\eta, x_{1}, x_{2}\right)$ must asymptotically converge to the origin and the parameter estimate $\hat{p}$ is bounded on $[0, \infty)$. This fact can be verified from Figure 1, which plots the trajectories of these dynamic signals $\left(\eta(t), x_{1}(t), x_{2}(t), \widehat{p}(t)\right)$. It can be seen that, at about $t=4.17 \mathrm{~s}, t=0.68 \mathrm{~s}$, and $t=0.59 \mathrm{~s}$, the states approach the origin, and at $t=0.36 \mathrm{~s}$, the parameter estimate $\widehat{p}(t)$ is bounded near $p^{*}=1.1$. In addition, according to Theorem 11, the control input $u$ is convergent to the origin. Figure 2 demonstrates this result, and it can be shown that, at about $t=0.51 \mathrm{~s}$, the input signal $u$ approaches the origin. As can be seen from Figures 1 and 2, our control scheme provides a fairly good asymptotic stabilization performance.

\section{Conclusion}

The state feedback stabilization problem is investigated for a class of nonlinear systems with dynamic uncertainties and uncertain control coefficients in this paper. The dynamic uncertainty is characterized by the uncertain ISS supply rates. A global asymptotic stabilization control scheme is proposed using the backstepping design scheme. The tuning function technique is applied in this procedure, which avoids the disadvantage of overparameterization. It is shown that, under some more restrictive conditions, a linear state feedback controller can be designed by the presented algorithm. The simulation example demonstrates the effectiveness of the proposed method.

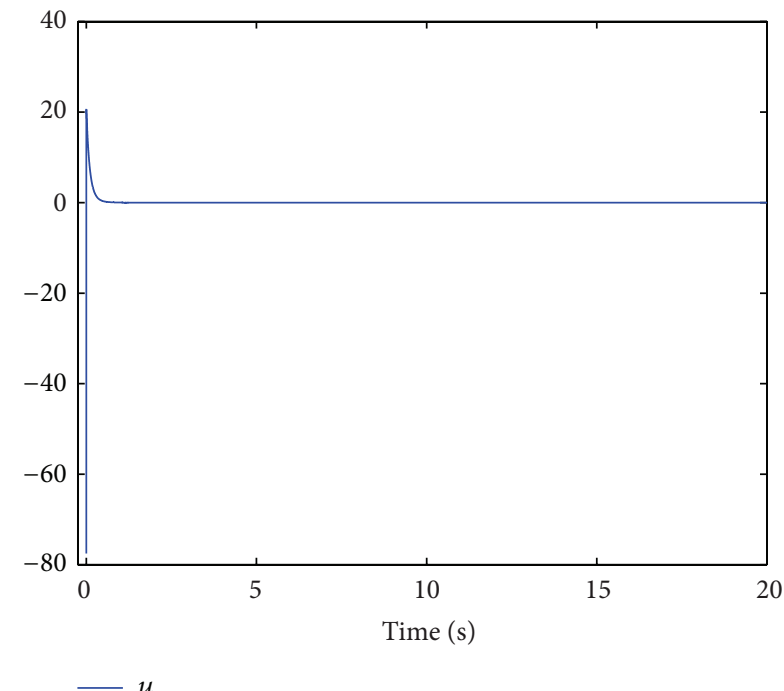

FIgURE 2: The control input of the closed-loop system.

\section{Conflict of Interests}

The authors declare that there is no conflict of interests regarding the publication of this paper.

\section{Acknowledgments}

This work is supported in part by the National Natural Science Foundation of China under Grant no. 61304008, the Shandong Provincial Natural Science Foundation of China under Grant no. ZR2013FQ033, and the Doctor Research Foundation of Shandong Jianzhu University under Grant no. XNBS1272.

\section{References}

[1] I. R. Petersen and R. Tempo, "Robust control of uncertain systems: classical results and recent developments," Automatica, vol. 50, no. 5, pp. 1315-1335, 2014.

[2] E. D. Sontag, "Smooth stabilization implies coprime factorization," IEEE Transactions on Automatic Control, vol. 34, no. 4, pp. 435-443, 1989.

[3] E. D. Sontag, "Comments on integral variants of ISS," Systems \& Control Letters, vol. 34, no. 1-2, pp. 93-100, 1998.

[4] P. Kokotovic and M. Arcak, "Constructive nonlinear control: a historical perspective," Automatica, vol. 37, no. 5, pp. 637-662, 2001.

[5] Z.-P. Jiang, A. R. Teel, and L. Praly, "Small-gain theorem for ISS systems and applications," Mathematics of Control, Signals, and Systems, vol. 7, no. 2, pp. 95-120, 1994.

[6] J. Tian, W. Feng, and Y. Wang, "High-order stochastic adaptive controller design with application to mechanical system," Mathematical Problems in Engineering, vol. 2012, Article ID 718913, 17 pages, 2012.

[7] N. Duan and H.-K. Liu, "Adaptive output feedback control for a class of stochastic nonlinear systems with SiISS inverse dynamics," Mathematical Problems in Engineering, vol. 2012, Article ID 673878, 15 pages, 2012. 
[8] Z.-P. Jiang, I. Mareels, D. J. Hill, and J. Huang, "A unifying framework for global regulation via nonlinear output feedback: from ISS to iISS," IEEE Transactions on Automatic Control, vol. 49, no. 4, pp. 549-562, 2004.

[9] Y. G. Liu, "Global stabilization by output feedback for a class of nonlinear systems with uncertain control coefficients and unmeasured states dependent growth," Science in China Series F: Information Sciences, vol. 51, no. 10, pp. 1508-1520, 2008.

[10] X. Yan and Y. Liu, "Global practical tracking for high-order uncertain nonlinear systems with unknown control directions," SIAM Journal on Control and Optimization, vol. 48, no. 7, pp. 4453-4473, 2010.

[11] Y.-Q. Wu, J.-B. Yu, and Y. Zhao, "Further results on global asymptotic regulation control for a class of nonlinear systems with iISS inverse dynamics," IEEE Transactions on Automatic Control, vol. 56, no. 4, pp. 941-946, 2011.

[12] D. Xu and J. Huang, "Output regulation for output feedback systems with iISS inverse dynamics," Journal of Dynamic Systems, Measurement and Control, Transactions of the ASME, vol. 133, no. 4, Article ID 044503, 2011.

[13] X. Yu, Y.-Q. Wu, and X.-J. Xie, "Reduced-order observer-based output feedback regulation for a class of nonlinear systems with iISS inverse dynamics," International Journal of Control, vol. 85, no. 12, pp. 1942-1951, 2012.

[14] X. Yu and G. Liu, "Output feedback control of nonlinear systems with uncertain ISS/iISS supply rates and noises," Nonlinear Analysis. Modelling and Control, vol. 19, no. 2, pp. 286-299, 2014.

[15] J. Yu and Y. Wu, "Global set-point tracking control for a class of non-linear systems and its application in continuously stirred tank reactor systems," IET Control Theory \& Applications, vol. 6, no. 12, pp. 1965-1971, 2012.

[16] Y.-G. Liu, "Output-feedback adaptive control for a class of nonlinear systems with unknown control directions," Acta Automatica Sinica, vol. 33, no. 12, pp. 1306-1312, 2007.

[17] F. Hong, S. S. Ge, B. Ren, and T. H. Lee, "Robust adaptive control for a class of uncertain strict-feedback nonlinear systems," International Journal of Robust and Nonlinear Control, vol. 19, no. 7, pp. 746-767, 2009.

[18] M. Krstić, I. Kanellakopoulos, and P. V. Kokotović, Nonlinear and Adaptive Control Design, Wiley-Interscience, New York, NY, USA, 1995.

[19] E. Sontag and A. Teel, "Changing supply functions in input/state stable systems," IEEE Transactions on Automatic Control, vol. 40, no. 8, pp. 1476-1478, 1995.

[20] W. Qiang-de, W. Chun-ling, and W. Yu-qiang, "Adaptive control of stochastic nonlinear systems with uncontrollable linearization," International Journal of Adaptive Control and Signal Processing, vol. 23, no. 7, pp. 667-678, 2009.

[21] H. K. Khalil, Nonlinear Systems, Prentice Hall, Upper Saddle River, NJ, USA, 2002. 


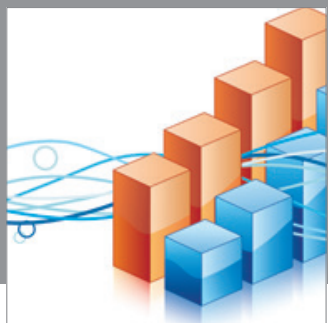

Advances in

Operations Research

mansans

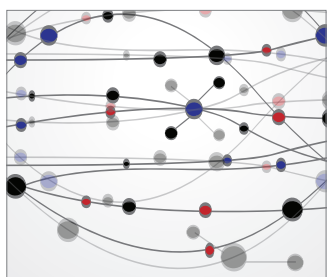

The Scientific World Journal
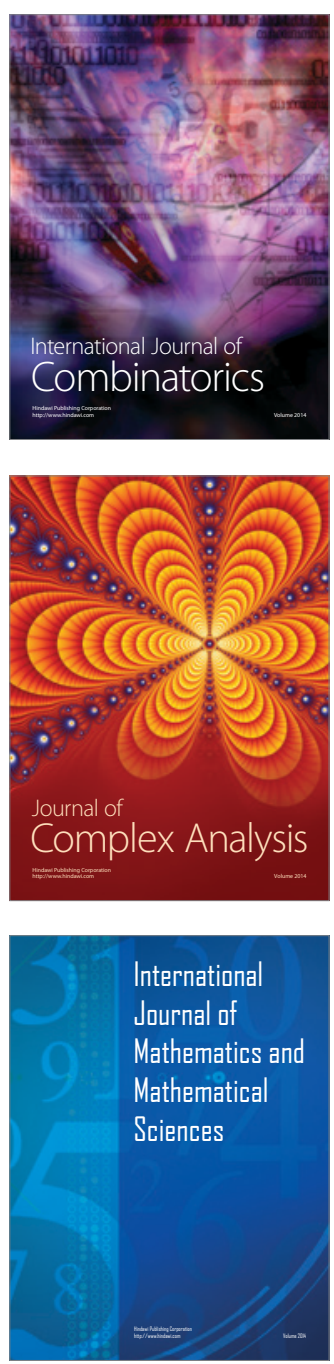
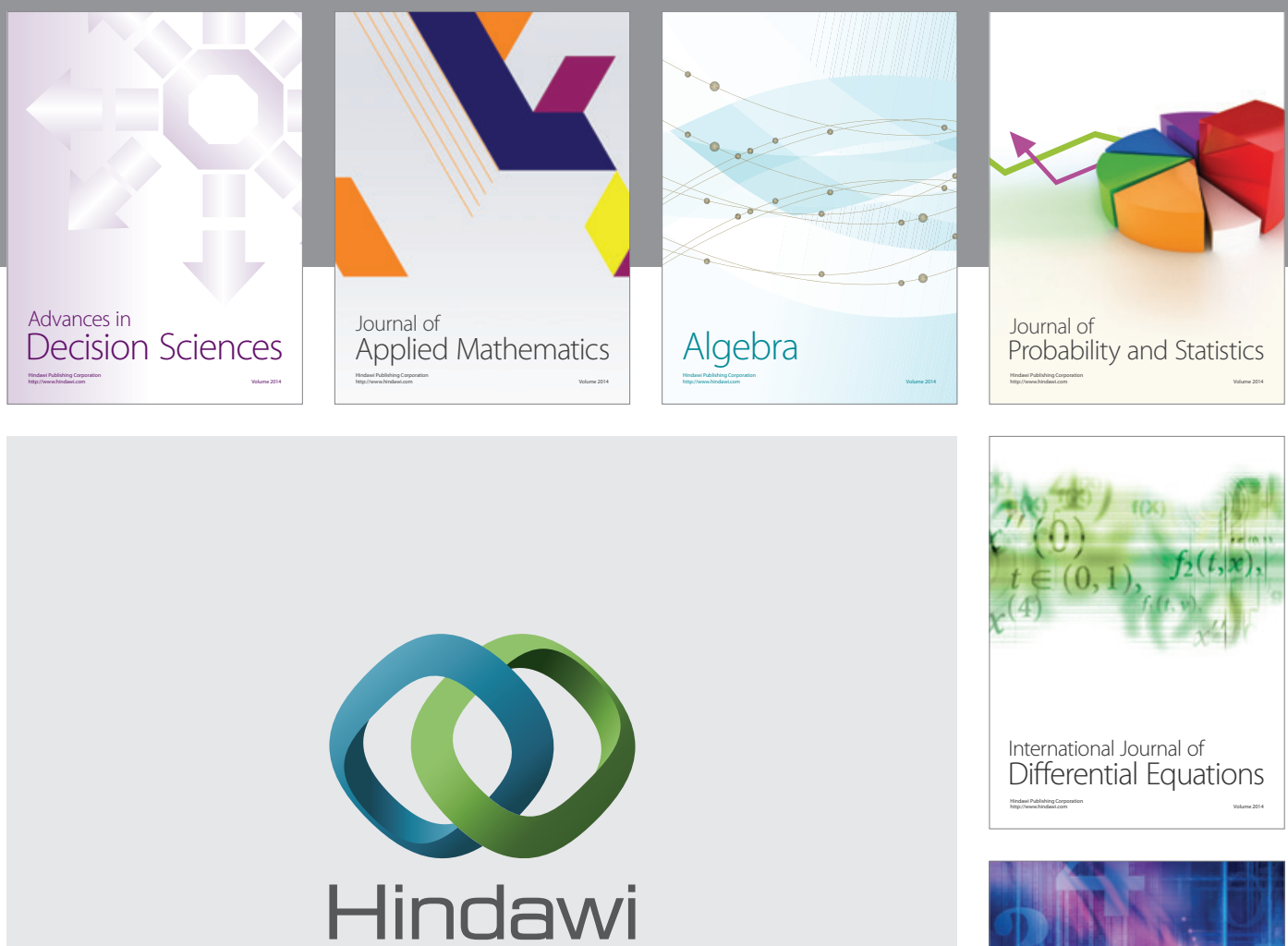

Submit your manuscripts at http://www.hindawi.com
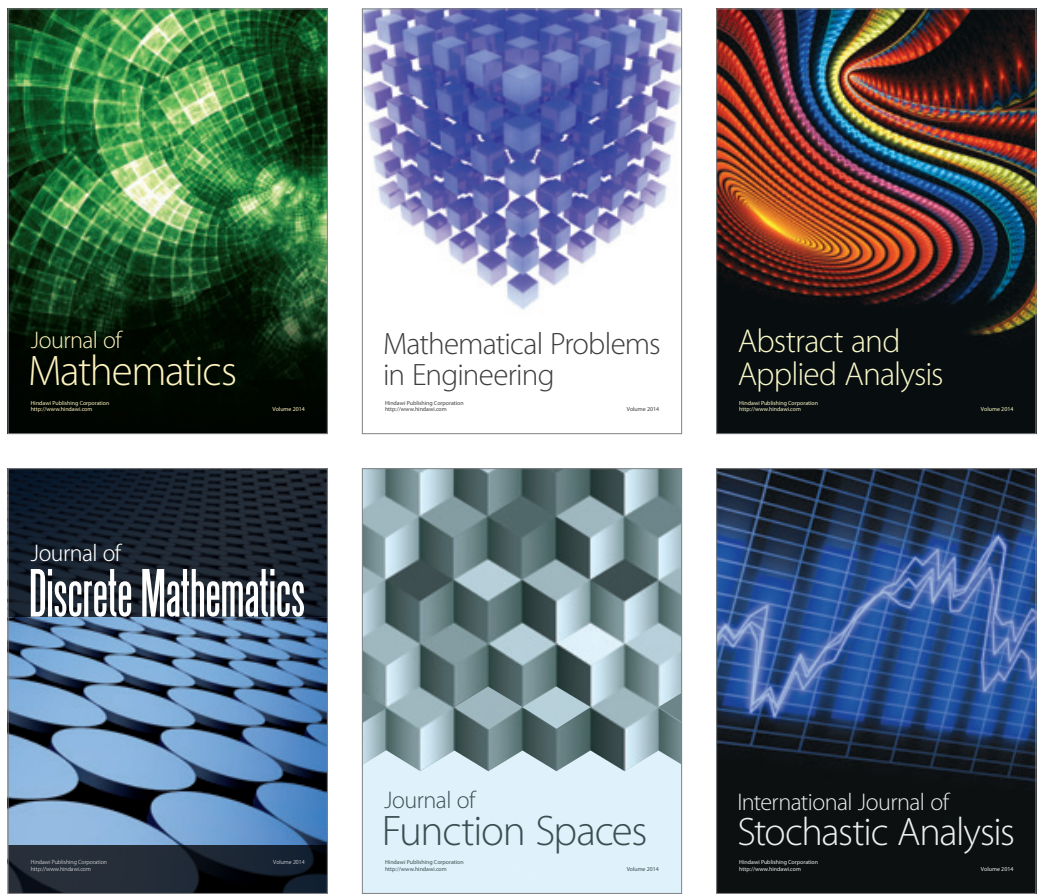

Journal of

Function Spaces

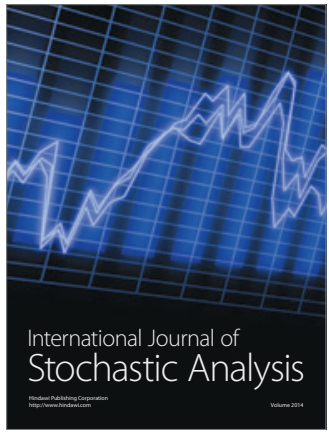

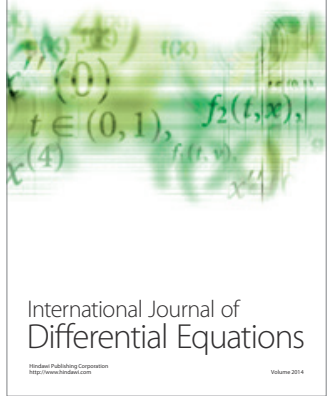
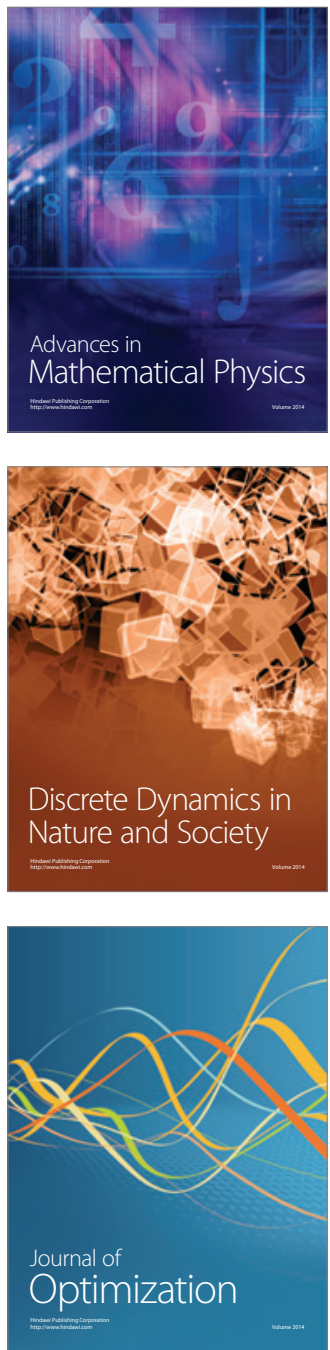